\title{
The Numerical Simulation of High Aspect Ratio Vertical SLAT Type Otter Board
}

\author{
Junting Yuan, Junchi Ma, Qi Li, Xuchang Ye \& Shiming Wang \\ College of Engineering Science and Technology Shanghai Ocean University, Shanghai, 201306, China
}

\begin{abstract}
Fluid simulation software FLUENT was used to study the flow distribution of vertical SLAT type otter board, when the SLAT angle $(\theta)$ ranging from 28 to 40 degree, and analyzed the relationship between SLAT angle and lift coefficient, drag coefficient, lift-to-drag ratio. Simulated the flow distribution in different attack angles $(\alpha)$ when the velocity at $1.54 \mathrm{~m} / \mathrm{s}$, analyzed the relationship between attack angles and lift coefficient, drag coefficient, lift-to-drag ratio. Also simulated the dynamics parameters when the velocity changing from 0.51 to $2.57 \mathrm{~m} / \mathrm{s}$, analyzed the relationship between Reynolds and lift coefficient, drag coefficient, lift-to-drag ratio. Aimed at provided theoretical help for the design and improvement of the otter board. KEYWORD: vertical SLAT type otter board; numerical simulation; hydrodynamic performance
\end{abstract}

\section{INTRODUCTION}

Single-boat trawling is an efficient, proactive and flexible way of fishing, which is one of the most important practices of world's marine fisheries. Otter board is main trawl accessories, to designing and producing good performance otter board not only affect the fishery harvesting and economic benefit of the single boat trawl but also eco-energy of trawling. Fisheries developed foreign countries have developed varieties of high-performance otter board which used in large pelagic single-boat trawling, broadly divided into the following forms, vertical Vtype curved otter board, rectangular V-type curved otter board, curved vertical SLAT otter board, vertical V-type double-wing otter board, large slotted otter board, high-performance jet otter board etc. Because of the domestic offshore trawling ship types and power, nets sizes are different, and when designing and using otter board have not in view of the type, it is effected on harvesting benefit, energy saving and consumption reducing. Besides, high seas large-scale trawling is most dependent on foreign designed or improved, it is impacted on improvement of nets. Therefore, it is necessary to researched on hydrodynamic performance of the foreign advanced otter board, and offered help to the design and improvement of the otter board, and realized the localization of the otter board (Helm J D et al, 2003).

Scholars at home and abroad did many studies on the hydrodynamic performance of various otter boards through the method of model experiment re- search. The Japanese scholars studied hydrodynamic performance of the double-wing otter board in different aspect ratio and guide plate curvature, concluded that rectangular "V" style otter board is better than the rectangle (Wei Z G et al, 2011) (Pan B et al, 2009). Domestic scholars $\mathrm{Xu}$ Baosheng etc did the production test and drew a conclusion that rectangular "V" style curved otter board is better than the "V" style otter board in the expansivity and efficiency. Guan Changtao and $\mathrm{Xu}$ Baosheng etc did the pneumatic model experiment on performance of the foreign advanced otter board and discovered that the high aspect ratio vertical type otter board is optimal in the comprehensive performance (Schreier $\mathrm{H}$ W et al, 2004) (Sutton M A et al, 2008), of which Guan Changtao researched the high aspect ratio vertical SLAT type otter board (Sutton M A et al, 2008). But almost all of the research is done through the sink or wind tunnel experiments, the experimental method is complex and the manpower cost huge. Computational fluid dynamics numerical simulation method which arose in the last century 60s to quantitatively measured the flow field numerical solution, the paper concluded flow field of high aspect ratio vertical SLAT type otter board by this method (Larsson L et al, 2004).

High aspect ratio vertical SLAT type otter board is widely used in the middle or bottom single boat trawl, it has the advantages of well expansivity, wide range of the attack angle and stable dragging, aspect ratio is generally greater than 1.0 while the larger is above 2.0. 
The physical model used in the research is high aspect ratio vertical SLAT type otter board which designed by East China Sea China Fisheries Research Institute(Fig.1). The basic parameters for the otter board as bellow, wing span (l) length is 6065 $\mathrm{mm}$, chord (b) length is $1845 \mathrm{~mm}$, aspect ratio $(\lambda)$ is 2.745 , seam width is $245 \mathrm{~mm}$, thickness is $30 \mathrm{~mm}$, weighs is $3267 \mathrm{~kg}$.
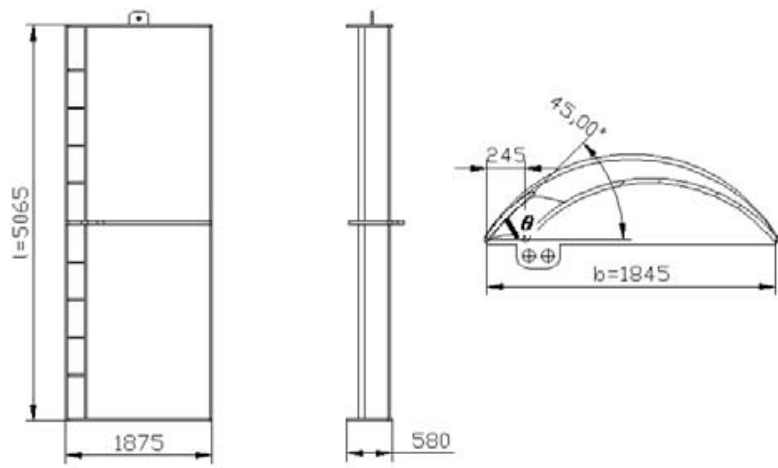

Fig.1 Sketch map of the otter board

\section{NUMERICAL SIMULATION STUDIES}

Analyzed the lift and drag coefficient (Cl\&Cd) and lift-to-drag ratio $(\mathrm{K})$ while SLAT angle $(\theta)$ ranging from 28 to 40 degree, stream speed is $1.54 \mathrm{~m} / \mathrm{s}$ (towing speed is $3 \mathrm{kn}$ ), attack angle is 20 degree. Analyzed the hydrodynamic performance of otter board under different attack angles $(\alpha)$ while stream speed is $1.54 \mathrm{~m} / \mathrm{s}$. Analysis the effect of Reynolds on the otter board while attack angle is 20 degree.

\section{FLUENT}

Computational fluid dynamics mathematical model is a series of partial differential equations. Its basic way is discretizing the fluid region, establishing the algebraic equations based on the certain principles, then solving the algebraic equations and getting the approximate solution of unknown variables. FLUENT software is adopted in the finite volume method, which is dividing the computational domain into a series of control volume, and each control volume has a node as representative, getting integral of the control equation and exporting the discrete equations (Larsson L et al, 2004).

\section{CALCULATION MODEL OF OTTER BOARD}

\subsection{Established physical model of otter board}

The 3D model of otter board with the proportion of 1:1 was established in SolidWorks. The Q345B high-tensile structural steel was used and the .x_t format was exported what can be read by Gambit.

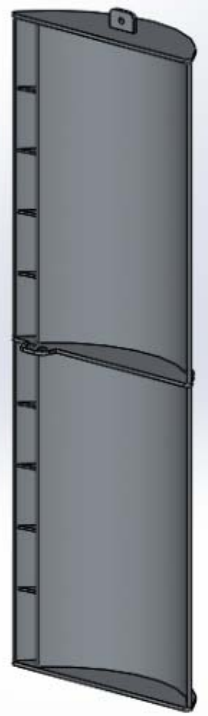

Fig.2.1 Solidworks 3D model

\subsection{The grid division and boundary definition}

Usually the flume experiment is used to analysis the state of the flow field of otter board in the water. The flume model is established to analysis the the external flow problem during numerical simulation. Flume boundary should be far away from the otter board, however that is impossible for simulation, so usually the boundary is setted for a distance as 10 times as the length of wing chord. The flume was established at the size of $4 \times 8 \times 10\left(\mathrm{~m}^{3}\right)$. Boolean subtraction operation wae used to subtract volume of otter board from flume, the results obtained is the part that required for solution. As the method of regionvolume-meshing $0.1 \mathrm{~m}$ interval size was used near the otter board and $0.2 \mathrm{~m}$ interval size was used in the rest. Using this method can make the grid close to the otter board dense and then solution precision can be improved. Tet/Hybrid from Elements was chosen as the type of grid division, which grid is mainly including tetra, then hexahedral grid, cone and wedge in the appropriate position. TGrid was chosen as the method of grid division to divide body into tetra units, then hexahedral, cone and wedge in the appropriate position. The inlet boundary was set as VELOCITY_INLET, flued velocity was constant, and the velocity magnitude was $1.54(\mathrm{~m} / \mathrm{s})$, outlet boundary used OUTFLOW, otter board surface used WALL boundary conditions, that was no smooth moving boundary conditions by default, NearWALL Treatment used Standard WALL Functions, the rest was WALL by default, Operating Conditions was atmospheric pressure 101325 PASCAL by default (Larsson L et al, 2004) (Zhou Z B et al, 2011). 


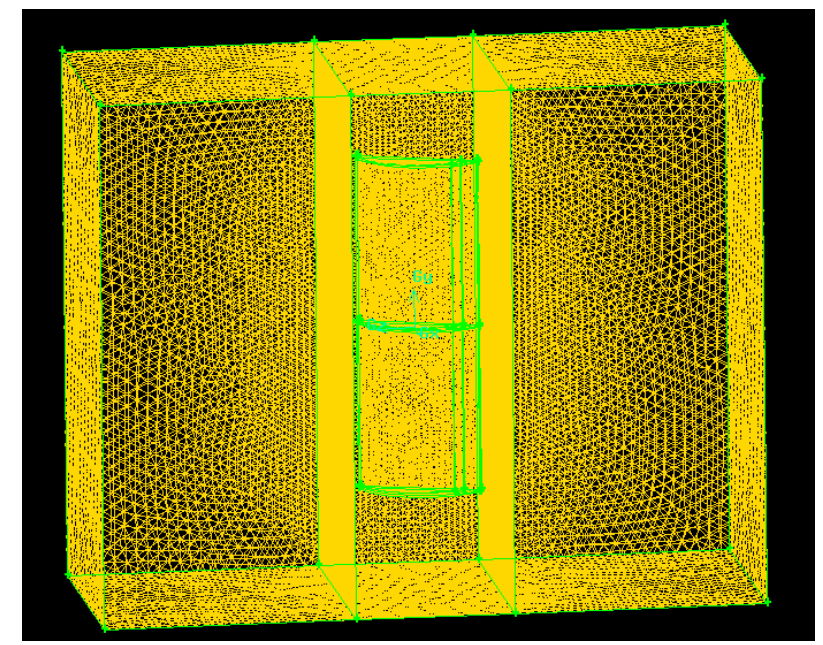

Fig.2.2 Solve model of flume

\subsection{Solver selection and Settings}

The segregated solver what was suitable for the incompressible or micro-compressible fluid was used to solved these case. Turbulence viscosity coefficient method (vortex model) was chosen from Reynolds as the turbulence numerical simulation method. The standard $k-\varepsilon$ model was used to calculated the turbulent stress by isotropic turbulence dynamic viscosity. The standard SIMPLE algorithm was used in pressure-velocity coupling. Second Order Upwind was used in the discrete format, which is mainly suitable for $3 \mathrm{~d}$ tetrahedron grid and complex flow.

\section{RESULTS AND DISCUSSION}

Analyzed relationship between SLAT angle and lift coefficient, drag coefficient, lift-to-drag ratio at attack angle is 20 degree at inflow velocity is $1.54 \mathrm{~m} / \mathrm{s}$ when SLAT angle $(\alpha)$ changing. Analyzed flow field at inflow velocity is $1.54 \mathrm{~m} / \mathrm{s}$ at SLAT angle is 35 degree when attack angle $(\alpha)$ is changing. Analyzed relationship between Reynolds and lift coefficient $(\mathrm{Cl})$, drag coefficient (Cd), lift-to-drag ratio (K) at attack angle is 20 degree when inflow velocity is changing.

\subsection{Flow regime analysis of changes SLAT angles}

Fig.3.1 is the contours of static pressure in cross section of otter board at the $1.2 \mathrm{~m}$ depth of flume under different SLAT angles $(\theta)$, it is observed that the pressure field distribution of otter board was consistent, the upwind side (Fig.3.1 right) was positive pressure and the downwind side (Fig.3.1 left) was negative pressure. There was pressure difference between them, which is help otter board expand outward. The pressure at the leading edge (Fig.3.1 down) of the upwind side was low and the trailing edge (Fig.3.1 up) was high while the pressure at the leading edge of the downwind side was high and that at the trailing edge was low. The pressure difference between the upwind side and the downwind generated torque around the $\mathrm{Z}$ axis. This torque could adjust the expansion range of otter board cooperated with different hole position of wrap board (Meng L B et al, 2007).
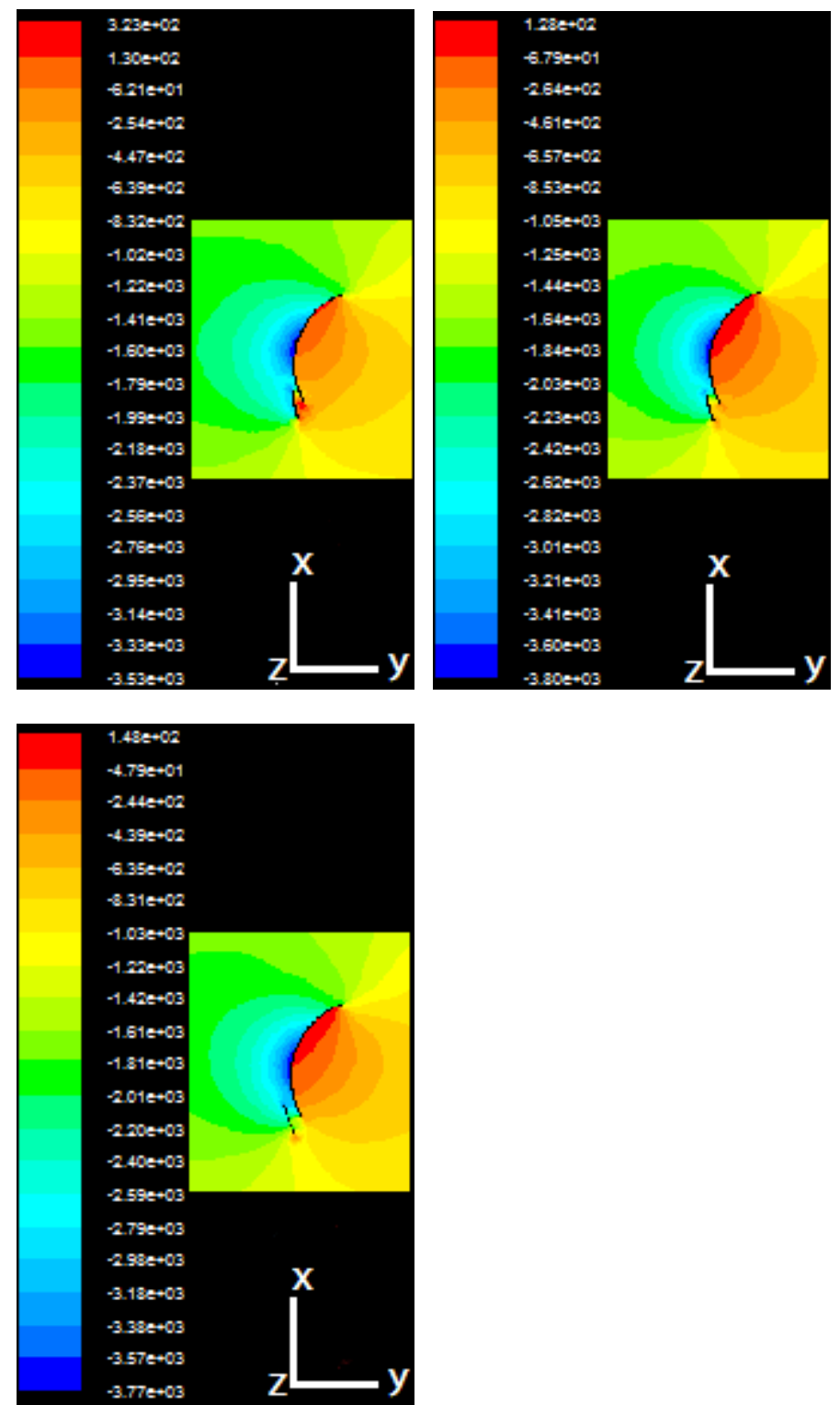

Fig.3.1 Contours of Static Pressure (pascal)

\subsection{Force analysis of changes SLAT angles}

While the SLAT angle ranged from 28 to 40 degree, lift (R1) and drag (Rd) was read from "Report" menu in FLUENT software. The lift and drag coefficient of otter board under different SLAT angles were solved according to the lift coefficient formula (1) and the drag coefficient formula (2), then solved lift-to-drag ratio according to the formula 3, where " $\rho$ " represents the density of water, " $s$ " represents the area of otter board and " $v$ " represents the inflow velocity. Figure 3.2 is curve of SLAT angle $(\theta)$ and lift-to-drag ratio $\mathrm{K}$ of otter board under different 
SLAT angles $(\theta)$.

$$
\begin{aligned}
& C_{l}=\frac{2 R_{l}}{\rho s v^{2}} \\
& C_{d}=\frac{2 R_{d}}{\rho s v^{2}} \\
& K=\frac{C_{l}}{C_{d}}
\end{aligned}
$$

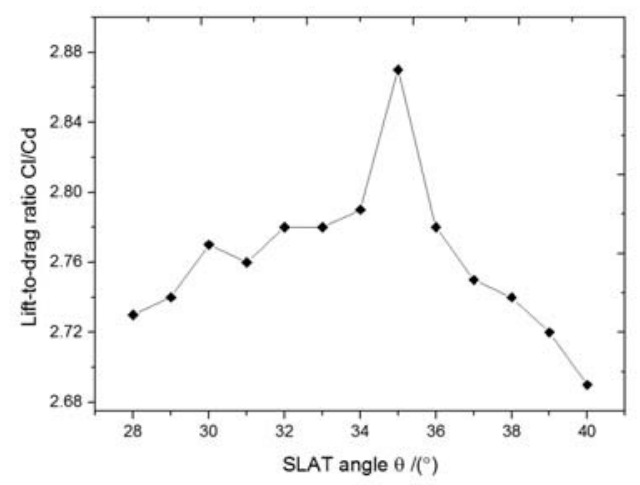

Fig.3.2a Curve of SLAT angle and lift-to-drag ratio

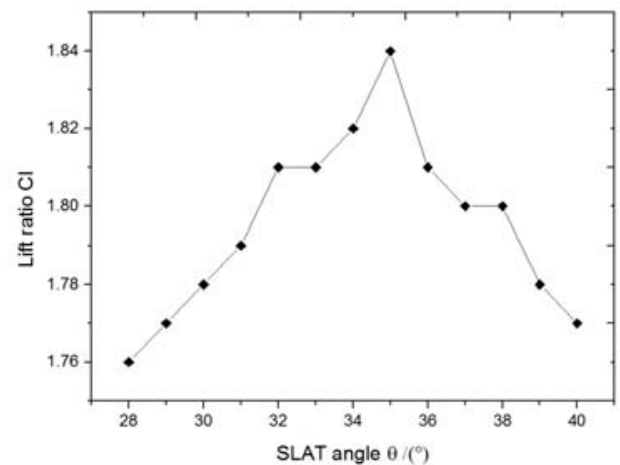

Fig.3.2b Curve of SLAT angle and lift coefficient

Figure 3.2a is the curve of SLAT angle $(\theta)$ and lift-to-drag ratio $(\mathrm{K})$. It can be seen that lift-to-drag ratio increased when $\theta$ increasing from 28 to 35 degree, and $\mathrm{K}$ reached the maximum value approximately 2.87 when $\theta$ is 35 degree. As SLAT angle $(\theta)$ increased lift-to-drag $(\mathrm{K})$ decreased gradually when $\theta$ is greater than 35 degree. Also it can be seen that the value of lift-to-drag ratio $(\mathrm{K})$ approximately 2.77 to 2.79 when $\theta$ ranging from 30 to 34 degree, indicated that otter board could keep high efficiency in this range, while curve of SLAT angle and lift coefficient dropped rapidly when $\theta$ is greater than 35 degree, indicated that efficiency of otter board dropped rapidly when $\theta$ at a high value. Still it can be seen that the value of lift-to-drag ratio (K) approximately 2.69 to 2.87 when $\theta$ ranging from 28 to 40 degree, indicated that otter board could keep high efficiency when SLAT angle of the commonly used range. Figure $3.2 \mathrm{~b}$ is curve of SLAT angle and lift coefficient, basically accorded with the curve of the curve of SLAT angle $(\theta)$ and lift-todrag ratio $(\mathrm{K})$, although lift coefficient appeared a certain fluctuation within the range from 30 to 34 degree, but the overall is still keep rising trend, and $\mathrm{K}$ reached the maximum value approximately 1.84 when $\theta$ is 35 degree.

\subsection{Hydrodynamic performance analysis of changes attack angles}

We often use lift-to-drag ratio which is the ratio of lift coefficient and drag coefficient to evaluate the expansion performance of otter board, the greater the ratio the greater the lift and the smaller of drag, also the efficiency of otter board is higher. Therefore lift-to-drag ratio is one of the main evaluation index of evaluating otter board performance. Observed the change of the pressure field at inflow velocity is $1.54 \mathrm{~m} / \mathrm{s}$ at SLAT angle is 35 degree when changing attack angles. Figure 3.3 is curve of attack angle and lift coefficient, drag coefficient, lift-to-drag ratio when the inflow velocity and SLAT angle is constant. It can be seen that lift coefficient and drag coefficient showed a trend of increasing when the attack angle within the range from 0 to 35 degree, but the changes of the lift-to-drag ratio is different, the lift-to-drag ratio increased when attack angle in the range from 0 to 10 degree, and lift-to-drag ratio reached an extremum value when the attack angle is 10 degree, and the lift-to-drag ratio showed a trend of decrease within the range from 10 to 35 degree, indicated that the performance of otter board is when attack angle is 10 degree. Values of curve kept between 2.77 and 2.87 when attack angles within the range from 5 to 20 degree, that is to say the SLAT otter boards has a wide range of the attack angle compared with other otter boards (Sutton M A et al, 2008).

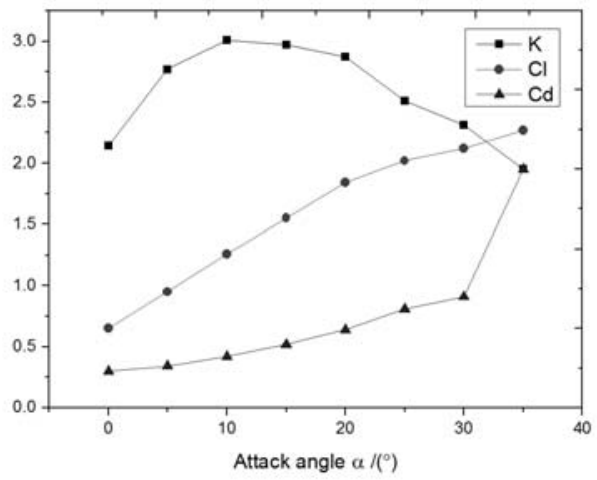

Fig.3.3 Curve of attack angle and lift coefficient, drag coefficient, lift-to-drag ratio

\subsection{The influence of Reynolds on the state of flow field around the otter board}

To Study the numerical simulation of otter board belongs to the classic case that is flow around cylinder in CFD, Reynolds plays a decisive role in flow around cylinder case, with the increase of Reynolds, and the viscous incompressible fluid flow around the 
cylinder can appear a variety of different flow state. In the small Reynolds number, flow is constant. With the increase of Reynolds number, a pile of trailing vortex will be shown after the cylinder. When Reynolds number is larger, the instability first appear in the wake flue, and also appeared a periodic oscillation in it, and then attached vortex shedding in wake flue alternately, and formed the Karman vortex street. With the further increase of Reynolds number, flow is becoming more and more complicated. In practical calculation, we observe the change of Reynolds number by changing inflow velocity. Formula 4 is design formula of Reynolds when otter board by flow, where " $v$ " represents the inflow velocity, " $l$ " represents wing chord, and " $v$ " represents the kinematic viscosity. Reynolds was obtained by formula 4, and its value changed within the range from 938 to 4727, at this point Reynolds in SCHAZ, while boundary layer is laminar separation, and the wake was turbulent vortex street (Pan B et al, 2009).

$$
\operatorname{Re}=\frac{v l}{v}
$$

Figure 3.4 is curve of Reynolds and lift coefficient, drag coefficient, lift-to-drag when the Reynolds number, was 938, 1895, 2833, 3789, 4727 respectively. Can be seen from the diagram that the slope of the K-Recurve was greater when $R e<3000$, while curve slope was smaller when $R e>3000$, indicated that lift-to-drag ratio increased slowly when Reynolds number is too large. When Reynolds number increased within the range from 938 to 4727, $\mathrm{Cl}-\mathrm{Re}$ curve was increased quickly than $C d$ - Re curve, and the slope of $C l-R e$ curve increasing continually, indicated that increased the velocity have an obvious effect on improving the lift coefficient, we can improve the towing speed appropriately to increase the hydrodynamic performance of the otter board in practical application.

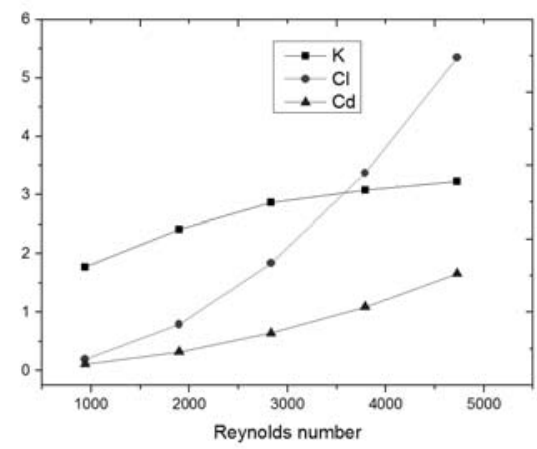

Fig.3.4 Curve of Reynolds and lift coefficient, drag coefficient, lift-to-drag ratio

\section{CONCLUSION}

Changing SLAT angle had an effect on the hydrodynamic performance of SLAT otter board. While the lift-drag ratio and lift coefficient of otter board were reached maximum value when SLAT is 35 degree, and the otter board had highest efficiency in this time.

SLAT otter board had a wide range of working attack angle, lift-to-drag ratio could kept a greater value when attack angles within the range from 5 to 20 degree, while the lift-to-drag ratio reached a maximum value when attack angle is 10 degree, and the hydrodynamic performance of the otter board was best at this time.

The boundary layer is laminar separation when otter board working in common towing speed, and the wake was turbulent vortex street. It could increase the hydrodynamic performance of the otter board when improved the towing speed appropriately.

\section{ACKNOWLEDGEMENTS}

[1] State Oceanic Administration in 2013 marine renewable energy projects (SHME2013JS01);

[2] Shanghai 2014 outstanding technical leader project (14XD1424300);

[3] Shanghai combining military and civilian projects "Large-scale self-powered ocean observational floats industrial demonstration and extension".

\section{REFERENCES}

Helm J D, Sutton M A, McNeill S R. Deformations in wide, center - notched, thin panel, part I: three dimensional shape and deformation measurements by computer vision[J]. Optical Engineering, 2003, 42(5): 1293-1305.

Larsson L, Sjodahl M, Thuvander F. Microscopic 3-D deformation field measurements using digital speckle photography[J]. Optics and Lasers in Engineering, 2004, 41: 767777.

Meng L B, Jin G C, Yao X F. Application of iteration and finite element smoothing technique for deformation and strain measurement of digital speckle correlation[J]. Optics and Lasers in Engineering, 2007, 45(1): 57-63.

Pan B, Asundi A, Xie H M, et al. Digital image correlation using iterative least squares and pointwise Ieast squares for deformation field and strain field measurements[J]. Optics and Lasers in Engineering, 2009, 47(7-8): 865-874.

Pan B, Xie H M, Yan L, et al. Accurate measurement of satellite antenna surface using 3D digital image correlation technique[J]. Strain, 2009, 45: 194-200.

Schreier H W, Garcia D, Sutton M A. Advances in light microscope stereo vision[J]. Society for Experimental Mechanics, 2004, 44(3): 278-287.

Sutton M A, Yan J H, Tiwari V. The effect of out-of-plane motion on $2 \mathrm{D}$ and $3 \mathrm{D}$ digital image correlation measurements[J]. Optics and Lasers in Engineering, 2008, 46(10): 746-757.

Wei Z G, Deng X M, Sutton M A., et al. Modeling of mixedmode crack growth in ductile thin sheets under combined inplane and out-of-plane loading. Engineering Fracture Mechanics, 2011, 78(17): 3082-3101. 
Zhou Z B, Chen P W, Huang F L, et al. Experimental study on the micro mechanical behavior of a PBX stimulant using SEM and digital image correlation method[J]. Optics and Lasers in Engineering, 2011, 49(3): 366-70. 